\title{
Applications of vibrational spectroscopy in the study of flavin-based photoactive proteins
}

\author{
Jiang $\mathrm{Li}^{\mathrm{a}, *}$ and Teizo Kitagawa ${ }^{\mathrm{b}}$ \\ ${ }^{\text {a }}$ Chemistry Department, Ohio State University, Columbus, OH, USA \\ ${ }^{\mathrm{b}}$ Picobiology Institute, Graduate School of Life Science, University of Hyogo, Hyogo, Japan
}

\begin{abstract}
Flavin cofactor is known to perform diverse biological functions. Recently, its role as a photoreceptor has been identified. So far, three classes of photoactive flavoproteins have been recognized: phototropin with LOV (Light, Oxygen and Voltage) domain, blue light sensory protein with BLUF (Blue Light sensing Using Flavin adenine dinucleotide) domain and photolyase/cryptochrome protein with PHR (Photolyase Homology Region) domain. Photochemistry of flavin is the key to unravel the reaction mechanisms of photoactive flavoproteins in their biological functions such as DNA repair or signal transduction. Vibrational (Infrared and Raman) spectroscopy is a useful and sensitive tool to investigate the photochemistry of flavin in protein environments and has significantly contributed to elucidate the reaction mechanisms of these photoactive proteins. This study will survey recent advances in vibrational spectroscopic studies on this topic and remaining questions to be answered.
\end{abstract}

Keywords: Flavin, flavoprotein, photochemistry, vibrational spectroscopy, infrared, Raman, photosensor, DNA repair

\section{Introduction}

Flavin, which constitutes of a group of compounds having the heteroaromatic ring system of 7,8 dimethylisoalloxazine, is contained in protein as a cofactor and plays critical roles in various important processes in cells [36,47]. The traditional biological function of flavin is of catalysis in redox reactions. It was found recently, however, that this cofactor also works as a photoreceptor to initiate the light-induced DNA repairs and signal transductions. As an isoalloxazine skeleton could not be synthesized in human cells, it is obtained from the diet through riboflavin (vitamin $\mathrm{B}_{2}$ ) to maintain lives. The structure of flavin and its atomic numbering are illustrated in Fig. 1(a), where riboflavin, flavin adenine dinucleotide (FAD) and flavin mononucleotide (FMN) reside. Flavin is an extremely versatile molecule with three redox states. Figure 1(b) shows the oxidized (quinoid), one electron reduced (semiquinoid), and two electron reduced (hydroquinoid) states of flavin and their physiological ionic states.

Among the photoactive flavoproteins, FAD was first identified as a catalytical cofactor of photolyase in 1987 - a repair enzyme of photo-damaged DNA [20]. Successively in 1995, FAD was discovered in cryptochrome (CRY), which has a sequence homologous to photolyase [29]. CRY serves as a blue-light

\footnotetext{
${ }^{*}$ Corresponding author: Dr. Jiang Li, Chemistry Department, Ohio State University, 191 West Woodruff Avenue, Columbus, OH 43210, USA. Tel.: +1 614292 1177; Fax: +1 614292 7557; E-mail: li.810@ osu.edu.
} 


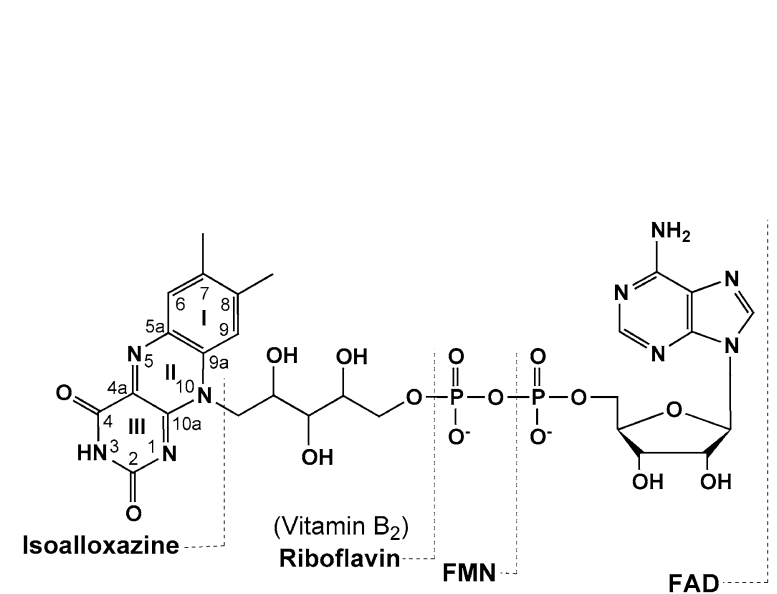

(a)

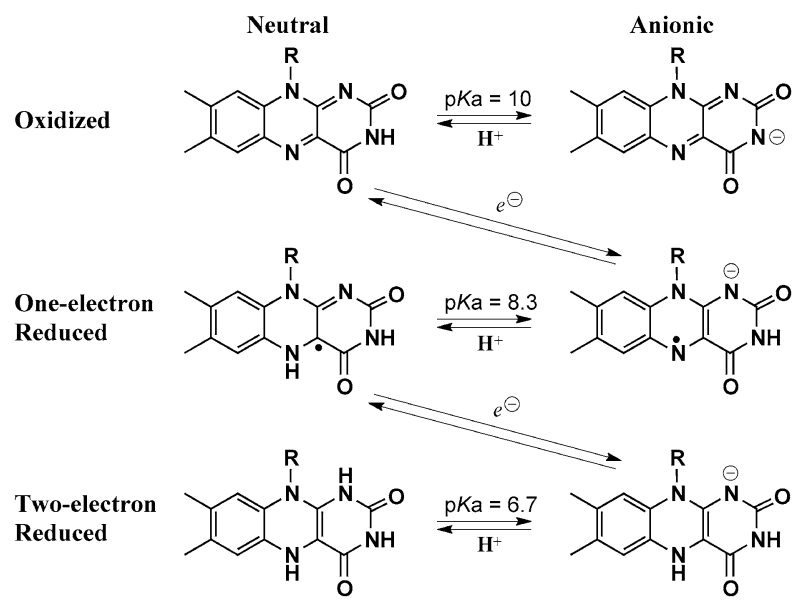

(b)

Fig. 1. (a) Structure of flavin derivatives (riboflavin, FMN, FAD) and the atomic numbering for isoalloxazine ring. (b) Redox and ionic states of flavin under physiological conditions.

receptor that regulates the light-induced responses of plants and also controls the circadian rhythm of animals. Recently, it was noted that CRY works as an important player in rapid light perception like rhodopsin [8]. In both photolyase and CRY, FAD is buried in a PHR domain. Secondly, phototropin, which regulates the phototropism responses in plants, came into the scene in 1997 [11]. Phototropin binds two FMN within a pair of LOV domains. At almost the same time, the third type of photoactive flavoproteins was found in blue light sensory proteins with BLUF domain in 1997 [13], and to date, six BLUF proteins have been characterized. So far, the DNA repair mechanism of FAD in PHR domain and the signal transduction mechanisms of FMN in LOV domain and of FAD in BLUF domain have been well explained. However, the photochemistry of FAD in the PHR domain of CRY is still elusive.

In the last decade, photochemistry of flavin in these photoactive proteins has been studied extensively with vibrational spectroscopy including Infrared (IR) and Raman spectra. The peak frequencies in vibrational spectra are discussed on the basis of the normal modes that are related to the strength of chemical bonds and effective masses of vibrating groups. Thus, the vibrational spectroscopy is a powerful tool to investigate structures of molecules. Here, we summarize the information provided to date on these photoactive flavoproteins by vibrational spectroscopy and discuss the remaining questions to be answered.

\section{FMN in LOV domain}

Phototropin contains two $\sim 12 \mathrm{kDa}$ FMN-binding domains, LOV1 and LOV2, at their N-terminus, and a serine/threonine kinase domain at the C-terminus [6]. As shown in Fig. 2(a), the oxidized FMN-bound LOV domain in the dark absorbs maximally around $450 \mathrm{~nm}$ (LOV 447) [6]. Blue light illumination triggers a photocycle involving the formation of the electronically excited triplet state of FMN, a reversible formation of a blue-shifted signaling state (LOV 370, absorbing maximally around $370 \mathrm{~nm}$ ), and a slowly restoration to the original LOV 447 state in the dark. The signaling state was demonstrated to involve a light-induced formation of a covalent bond between $\mathrm{C}(4 \mathrm{a})$ of flavin and a nearby cysteine (Fig. 2(a) right) [42]. Soon after this report, Swartz et al. presented the evidence for the formation of flavin C(4a)-thiol adduct using the Fourier Transform Infrared (FTIR) spectroscopy. The observed 


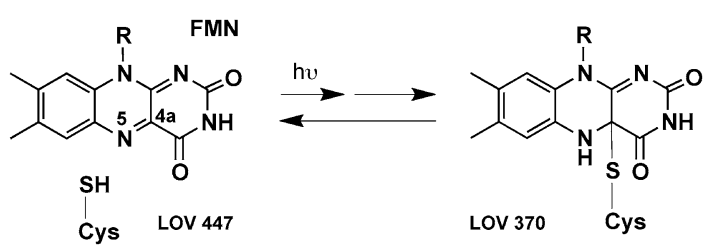

(a)

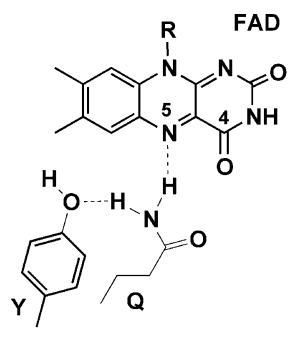

(b)

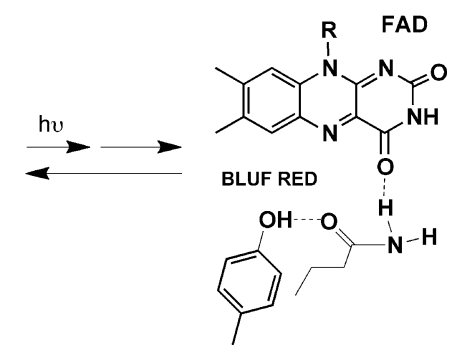

Fig. 2. Photochemistry of flavin. (a) In LOV domain, signaling state: LOV 370. (b) In BLUF domain, signaling state: BLUE RED.

spectra are consistent with the frequencies calculated for a flavin attached with a sulfur at the $\mathrm{C}(4 \mathrm{a})$ position [51]. The presence of $\mathrm{S}-\mathrm{H}$ groups in the dark state and its disappearance upon photoreaction was confirmed by IR spectroscopy [16]. More recently, a more comprehensive vibrational assignment of the flavin-thiol adduct was provided by Kikuchi et al. [21].

A formation mechanism of flavin-thiol adduct is one of the basic questions of LOV domain signaling. Phenomenologically, first a protonated cysteine (Cys57) near FMN was identified in the dark state LOV domain [4]. Second, a protonated cysteine and unprotonated flavin were detected for the excited triplet state of FMN by steady-state and time-resolved IR spectroscopy [1,40,43]. These results ruled out the ionic mechanism for the formation of flavin-thiol adduct suggested by other studies. Third, Alexandre et al. [3] found that the proximity of the cysteine to FMN not only enables the formation of a covalent adduct between flavin and cysteine, but also facilitates the rapid formation of the reactive flavin triplet state from their investigation of electronic structures and molecular vibrations of flavin. Interestingly, the formation of flavin-thiol adduct was engineered by introducing a cysteine residue into the BLUF domain, in which the occurrence of a LOV-like photoreaction of flavin was identified by FTIR and UV-vis spectroscopy, suggesting that the cysteine works as the key factor in determining the reaction of flavin in LOV domain [50]. Another basic question of LOV domain signaling is the signal transduction pathway. The conformational changes of protein induced by the $\mathrm{C}(4 \mathrm{a})-\mathrm{S}(\mathrm{Cys})$ bond formation were studied by IR spectroscopy, mostly by Kandori's group, who determined the key residues and protein fragment [2, $15,18,23,38,41,56,57]$. In addition, FTIR studies also revealed the $\mathrm{C}=\mathrm{O}$ stretching band of flavin [14] and the conformational heterogeneity of the LOV domain $[2,44]$. Although the photochemistry of flavin in the LOV domain has been well characterized, some questions remain unanswered. First, the mechanism by which the covalently bound flavin-thiol adduct relaxes to the initial dark state is still unknown. Second, the dynamics of the formation of flavin-thiol adduct has not been directly clarified.

\section{FAD in BLUF domain}

BLUF domain exhibits typical features of an oxidized FAD in the dark. Upon blue light illumination, the FAD forms an intermediate signaling state with a $\sim 10 \mathrm{~nm}$ red-shifted absorbance (BLUF RED) without apparent variations in the redox state of flavin. The red-shifted state of FAD returns to the original form in the dark. As shown in Fig. 2(b), it has been identified that the BLUF RED signaling state is formed when the hydrogen-bond between flavin and glutamine is switched from $\mathrm{N}(5)$ to $\mathrm{C}(4)=\mathrm{O}$ of flavin. Indeed, vibrational spectroscopic methods played an important role in clarifying this mechanism. 
Laan et al. [25] characterized the primary photochemistry of FAD in the BLUF domain using FTIR. The difference spectrum between dark and signaling states was dominated by a band shift from 1710 to $1698 \mathrm{~cm}^{-1}$ [25]. Such a large downshift as $\sim 15 \mathrm{~cm}^{-1}$ was observed by many successive IR and Raman studies and was assigned to the $\mathrm{C}(4)=\mathrm{O}$ stretching of the isoalloxazine ring (Fig. 1(a)) $[9,30,39,55]$. By examining the BLUF domain containing isotope-labeled FAD, Masuda et al. [33] revealed that the $\mathrm{C}(4)=\mathrm{O}$ stretching of isoalloxazine ring was the only band assignable exclusively to FAD, and that all the other bands came from protein moiety. The downshift indicates the formation of a strong H-bond at $\mathrm{C}(4)=\mathrm{O}$ when FAD is brought into the signaling state, which yielded the red-shifted absorption [10]. Unno et al. carried out Raman spectroscopic studies on wild-type and mutated BLUF domains, and clarified that the key mechanism is the H-bonding switch of Gln63- $\mathrm{NH}_{2}$ from $\mathrm{N}(5)$ to $\mathrm{C}(4)=\mathrm{O}$ of flavin as shown in Fig. 2(b) [54]. Iwata et al. [17] demonstrated that the switch is accompanied by the donation of H-bond from Tyr21-OH to Gln- $\mathrm{NH}_{2}$. The formation of the signaling state was further investigated by ultrafast IR spectroscopy [49]. On the other hand, Bonetti et al. observed the light-induced electron transfer from tyrosine to flavin through the formation of anionic semiquinoid flavin and a bleach of a vibrational mode of tyrosine, which suggests that the H-bond between glutamine and flavin is switched through a radical pair mechanism [5]. Other residues in signal transduction pathway also have been investigated $[24,31,32,53]$. Although the primary photochemistry of FAD in the BLUF domain has been well examined, the dynamics of structural changes around $\mathrm{N}(5)$ and $\mathrm{C}(4)=\mathrm{O}$ of flavin and more details of signal transduction pathway after light-excitation need to be clarified.

\section{FAD in PHR domain}

Photolyase is a blue-light activated enzyme that repairs ultraviolet (UV)-damaged DNA. There are two major UV-damaged DNA, cyclobutane pyrimidine dimer (CPD) and (6-4) photoproduct (64PP), which are repaired by CPD photolyase and (6-4) photolyase, respectively. The photo-excited FAD in photolyase adopts the anionic two-electron-reduced state (Fig. 1(b)) and splits the UV-damaged DNA dimer through a cyclic electron transfer between FAD and DNA. Schelvis and colleagues examined the CPD photolyase combined with DNA substrate by resonance Raman spectroscopy and discussed the vibrational modes of neutral one-electron-reduced FAD and their perturbations by substrate binding [37, 45]. On the other hand, FTIR spectroscopy was used by Schleicher et al. to monitor the vibronic bands of one- and two-electron-reduced FAD in this enzyme with and without substrate. Also, the characteristic bands of both FAD and DNA were assigned [46]. These studies provided the basis for understanding the structure and environment of FAD when placed in a PHR domain. For example, frequencies of bands between 1615 and $1150 \mathrm{~cm}^{-1}$ observed for neutral one-electron-reduced FAD are all lower in photolyase compared with those in neutral semiquinoid flavin in other flavoproteins, which suggests a stronger H-bonding environment in photolyase. In fact, the crystal structure of CPD photolyase has showed a very special U-shaped conformation of FAD, in which an adenine ring approaches the $\mathrm{N}(10)$ $\mathrm{C}(10 \mathrm{a})$ of the isoalloxazine ring (Fig. 3(a)) and an intramolecular H-bond is formed between the 2'-OH of the ribityl chain and the N(1) of isoalloxazine ring [35]. Such a bent FAD is unique in this protein family, but whether the adenine bridges the electron transfer between isoalloxazine and DNA substrate is still under debate.

Little was known about (6-4) photolayse when we first investigated this enzyme by resonance Raman spectroscopy [27,28]. We found that the H-D exchange at $\mathrm{N}(5)$ of isoalloxazine in a semiquinoid form was much more difficult compared with that at N(3). Complete H-D exchange was confirmed only when 

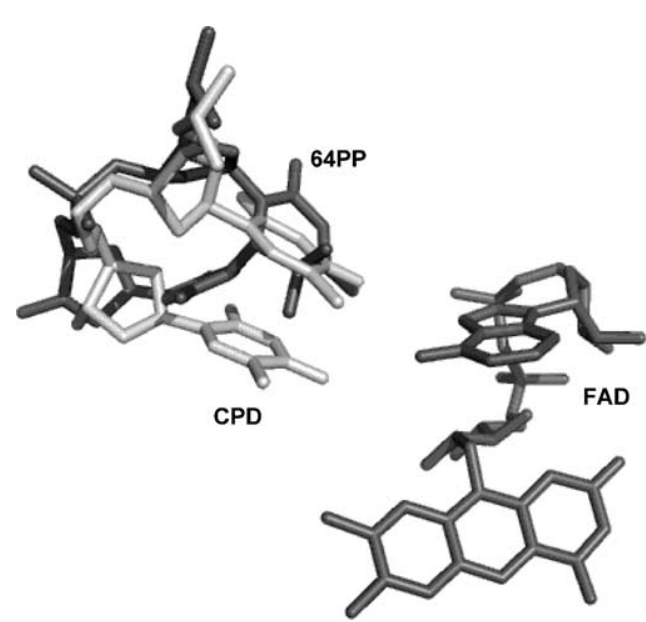

(a)

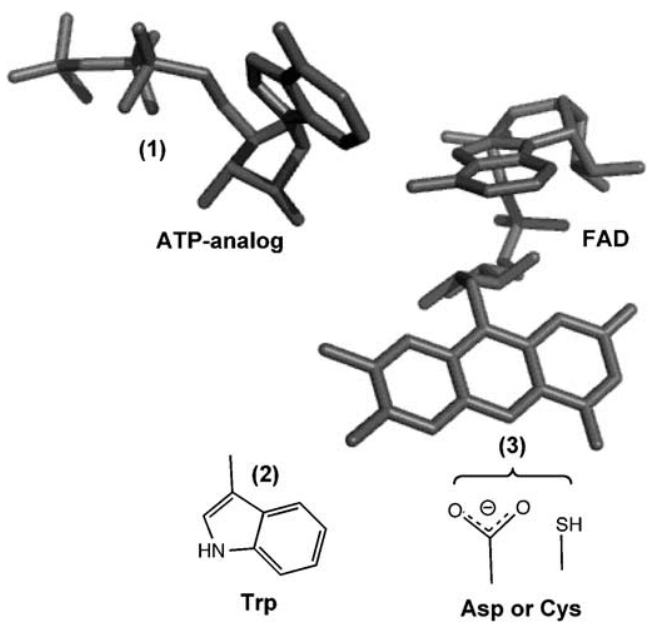

(b)

Fig. 3. Active sites of photolyase (a) and cryptochrome (b). (a) Structure of FAD and DNA substrates of 64PP and CPD (light-grey). (b) Structure of FAD and (1) the ATP in plant CRY that may accept an electron from light-excited flavin; (2) the Trp that donates an electron to flavin in the photoreduction; (3) residues (Asp in plant CRY, Cys in insect CRY) that determines the ionic state of semiquinoid flavin.

flavin was oxidized once and photoreduced to a semiquinone form again. Such a difficult $\mathrm{H}-\mathrm{D}$ exchange at $\mathrm{N}$ (5) of flavin was recognized also for CPD photolyase (unpublished data). Therefore, the assignments of vibrational bands involving the $\mathrm{N}(5)$ of flavin in this protein family by $\mathrm{H}-\mathrm{D}$ exchange $[22,45]$ have to be made with caution. With the help of the selectively deuterated isoalloxazines at N(3) or N(5), we assigned the observed Raman bands with the normal mode calculations for neutral semiquinoid flavin for the first time [27]. Based on this assignment and the Raman spectra of both (6-4) and CPD photolyases with and without DNA substrate, we suggested that the DNA disturbed the bent conformation of FAD in both enzymes, but only the ring I of isoalloxazine in CPD photolyase. 64PP was predicted to interact with adenine ring of FAD through $\mathrm{H}$-bonding (like CPD) but not to interact with isoalloxazine ring (unlike CPD) as shown in Fig. 3(a), and two years later this was, in fact, observed by X-ray crystallography [34]. Recently Li et al. observed that the electron transfer rate from FAD to substrate in (6-4) photolyase is similar to that in CPD photolyase [26]. All these results strongly suggest that the electron transfer from isoalloxazine to DNA substrate is bridged by adenine in both photolyase enzymes. In addition, a recent IR study on (6-4) photolyase noted that the substrate binding changed only the protein backbone and a protonated carboxylic acid side chain, whereas photoactivation yielded changes of FAD and surrounding protein [58]. It has been known that (6-4) photolyase is much flexible than CPD photolyase [7], and is highly homologous to the photoreceptor of CRY [52]. Thus, further investigations on the light-induced conformational changes of (6-4) photolyase may shed light on the molecular mechanism of CRY.

CRY is defined as photolyase homology without a DNA repair function and is classified into two groups: plant CRY and animal CRY. DASH-CRY was identified as a photolyase that repairs CPD in a single strand DNA. Resonance Raman spectroscopy has revealed that DASH-CRY has significant spectroscopic similarities to CPD photolyase [48]. The reaction mechanisms of plant and animal CRYs are better characterized in plant A. thaliana CRY (AtCRY) and insect D. melanogaster CRY (DmCRY), respectively. Upon irradiation with blue light, the conserved C-terminal extension domain undergoes a light-induced conformational change in plant and insect CRYs, which results in the modulations of 
downstream signaling partner proteins. Although the FAD cofactor in this protein must initiate the signal transduction, its catalytically active redox state in plant CRY is under debate. The semiquinoid anionic FAD was suggested as the signaling state in animal (insect) CRY, but the light-induced response of C-terminal extension has not been observed directly. In both CRYs, it is left to be solved what kind of photochemistry occurs to the flavin for initiating the conformational changes of protein. Figure $3(\mathrm{~b})$ shows several possible mechanisms that may disturb the protein structure of CRY: (1) photolyase-like electron transfer from flavin to an ATP bound in AtCRY1; (2) electron transfer from nearby conserved Trp to flavin in photoreduction reactions; (3) the changes of H-bonding or electrostatic interaction between N(5) of flavin and its nearby residues. Kottke et al. [22] observed deprotonation of an aspartic or glutamic acid upon illumination to AtCRY, which is probably the Asp near flavin (Fig. 3(b)). IR spectroscopy has revealed the changes in secondary structure of protein in response to the photoreduction of FAD [12,22] and noted a low frequency of the carbonyl stretch in plant CRY [12]. Interestingly, the conserved Cys near the N(5) of flavin in insect CRY stabilizes an anionic semiquinoid form of FAD, which is believed to be the signaling state. Such a stable anionic semiquinoid FAD was also observed in the Asp to Cys mutant of DASH CRY [19]. However, it is not clear why the Cys near flavin does not form the flavin-thiol adduct like the LOV domain and mutated BLUF domain. So far, the vibrational studies on animal CRY are lacking. Similar to the progresses in understranding LOV and BLUF domains, low temperature difference IR spectra before and after light illumination and site-directed mutagenesis as well as time-resolved techniques at room temperature are expected to clarify the photochemistry of FAD in CRY and to determine key residues in the signal transduction pathway.

\section{Concluding remarks}

Unlike the light-induced E/Z isomerization of photoactive cofactors in other biological photoreceptors such as retinoid proteins, phytochrome and photoactive yellow protein, flavin does not initiate the lightinduced signal transduction by a large structural change of itself. Vibrational spectroscopy is a powerful tool to detect the delicate conformational change of flavin and surrounding proteins and has indeed significantly contributed to the understanding of photochemistry in flavin-based photoactive proteins with LOV, BLUF and PHR domains. We believe that the recently-developed ultrafast time-resolved vibrational spectroscopy will unravel more details about the structural dynamics involved in initial signaling process, especially for the unknown photochemistry of FAD in PHR domain of CRY. Femtosecond IR spectroscopy has been applied to the studies on flavin-based photoactive proteins [1,5,49], but the spectral window was limited by the available probe frequencies and solvent absorption. Recently, femtosecond stimulated Raman spectroscopy (FSRR) has been successfully applied to the study of the photochemistry of bacteriorhodopsin, phytochrome, and green fluorescent protein. In contrast to femtosecond IR spectroscopy, FSRR can detect a wide UV-visible/near IR spectral region. In addition, resonance conditions can be chosen to selectively detect the specific ionic redox state of flavin without the influence of protein matrix. Therefore, FSRR may be a promising vibrational spectroscopic technique to investigate the mechanisms of flavin-based photoactive proteins in the future.

\section{Acknowledgements}

J. L. is supported by the Pelotonia Fellowship Program from the Ohio State University. T. K. is funded by the Global COE Program, "Picobiology: Life Science at the Atomic Level" at the Graduate School of Life Science, University of Hyogo from MEXT, Japan. 


\section{References}

[1] M.T. Alexandre, T. Domratcheva, C. Bonetti, L.J. van Wilderen, R. van Grondelle, M.L. Groot, K.J. Hellingwerf and J.T. Kennis, Primary reactions of the LOV2 domain of phototropin studied with ultrafast mid-infrared spectroscopy and quantum chemistry, Biophys. J. 97 (2009), 227-237.

[2] M.T. Alexandre, R. van Grondelle, K.J. Hellingwerf and J.T. Kennis, Conformational heterogeneity and propagation of structural changes in the LOV2/J $\alpha$ domain from Avena sativa phototropin 1 as recorded by temperature-dependent FTIR spectroscopy, Biophys. J. 97 (2009), 238-247.

[3] M.T. Alexandre, R. van Grondelle, K.J. Hellingwerf, B. Robert and J.T. Kennis, Perturbation of the ground-state electronic structure of FMN by the conserved cysteine in phototropin LOV2 domains, Phys. Chem. Chem. Phys. 10 (2008), 66936702.

[4] K. Ataka, P. Hegemann and J. Heberle, Vibrational spectroscopy of an algal Phot-LOV1 domain probes the molecular changes associated with blue-light reception, Biophys. J. 84 (2003), 466-474.

[5] C. Bonetti, T. Mathes, I.H. van Stokkum, K.M. Mullen, M.L. Groot, R. van Grondelle, P. Hegemann and J.T. Kennis, Hydrogen bond switching among flavin and amino acid side chains in the BLUF photoreceptor observed by ultrafast infrared spectroscopy, Biophys. J. 95 (2008), 4790-4802.

[6] W.R. Briggs and J.M. Christie, Phototropins 1 and 2: versatile plant blue-light receptors, Trends Plant Sci. 7 (2002), 204-210.

[7] C.W. Chang, L. Guo, Y.T. Kao, J. Li, C. Tan, T. Li, C. Saxena, Z. Liu, L. Wang, A. Sancar and D. Zhong, Ultrafast solvation dynamics at binding and active sites of photolyases, Proc. Natl. Acad. Sci. USA 107 (2010), 2914-2919.

[8] K.J. Fogle, K.G. Parson, N.A. Dahm and T.C. Holmes, Cryptochrome is a blue-light sensor that regulates neuronal firing rate, Science 331 (2011), 1409-1413.

[9] K. Hasegawa, S. Masuda and T.A. Ono, Structural intermediate in the photocycle of a BLUF (sensor of blue light using FAD) protein Slr1694 in a cyanobacterium synechocystis sp. PCC6803, Biochemistry 43 (2004), 14979-14986.

[10] K. Hasegawa, S. Masuda and T.A. Ono, Spectroscopic analysis of the dark relaxation process of a photocycle in a sensor of blue light using FAD (BLUF) protein Slr1694 of the cyanobacterium Synechocystis sp. PCC6803, Plant Cell Physiol. 46 (2005), 136-146.

[11] E. Huala, P.W. Oeller, E. Liscum, I.S. Han, E. Larsen and W.R. Briggs, Arabidopsis NPH1: a protein kinase with a putative redox-sensing domain, Science 278 (1997), 2120-2123.

[12] D. Immeln, R. Pokorny, E. Herman, J. Moldt, A. Batschauer and T. Kottke, Photoreaction of plant and DASH cryptochromes probed by infrared spectroscopy: the neutral radical state of flavoproteins, J. Phys. Chem. B 114 (2010), 17155-17161.

[13] M. Iseki, S. Matsunaga, A. Murakami, K. Ohno, K. Shiga, K. Yoshida, M. Sugai, T. Takahashi, T. Hori and M. Watanabe, A blue-light-activated adenylyl cyclase mediates photoavoidance in Euglena gracilis, Nature 415 (2002), 1047-1051.

[14] T. Iwata, D. Nozaki, Y. Sato, K. Sato, Y. Nishina, K. Shiga, S. Tokutomi and H. Kandori, Identification of the C=O stretching vibrations of FMN and peptide backbone by 13C-labeling of the LOV2 domain of Adiantum phytochrome3, Biochemistry 45 (2006), 15384-15391.

[15] T. Iwata, D. Nozaki, S. Tokutomi, T. Kagawa, M. Wada and H. Kandori, Light-induced structural changes in the LOV2 domain of Adiantum phytochrome3 studied by low-temperature FTIR and UV-visible spectroscopy, Biochemistry 42 (2003), 8183-8191.

[16] T. Iwata, S. Tokutomi and H. Kandori, Photoreaction of the cysteine S-H group in the LOV2 domain of Adiantum phytochrome3, J. Am. Chem. Soc. 124 (2002), 11840-11841.

[17] T. Iwata, A. Watanabe, M. Iseki, M. Watanabe and H. Kandori, Strong donation of the hydrogen bond of tyrosine during photoactivation of the BLUF domain, J. Phys. Chem. Lett. 2 (2011), 1015-1019.

[18] T. Iwata, A. Yamamoto, S. Tokutomi and H. Kandori, Hydration and temperature similarly affect light-induced protein structural changes in the chromophoric domain of phototropin, Biochemistry 46 (2007), 7016-7021.

[19] T. Iwata, Y. Zhang, K. Hitomi, E.D. Getzoff and H. Kandori, Key dynamics of conserved asparagine in a cryptochrome/photolyase family protein by Fourier transform infrared spectroscopy, Biochemistry 49 (2010), 8882-8891.

[20] M.S. Jorns, E.T. Baldwin, G.B. Sancar and A. Sancar, Action mechanism of Escherichia coli DNA photolyase. II. Role of the chromophores in catalysis, J. Biol. Chem. 262 (1987), 486-491.

[21] S. Kikuchi, M. Unno, K. Zikihara, S. Tokutomi and S. Yamauchi, Vibrational assignment of the flavin-cysteinyl adduct in a signaling state of the LOV domain in FKF1, J. Phys. Chem. B 113 (2009), 2913-2921.

[22] T. Kottke, A. Batschauer, M. Ahmad and J. Heberle, Blue-light-induced changes in Arabidopsis cryptochrome 1 probed by FTIR difference spectroscopy, Biochemistry 45 (2006), 2472-2479.

[23] T. Koyama, T. Iwata, A. Yamamoto, Y. Sato, D. Matsuoka, S. Tokutomi and H. Kandori, Different role of the J $\alpha$ helix in the light-induced activation of the LOV2 domains in various phototropins, Biochemistry 48 (2009), 7621-7628.

[24] W. Laan, M. Gauden, S. Yeremenko, R. van Grondelle, J.T. Kennis and K.J. Hellingwerf, On the mechanism of activation of the BLUF domain of AppA, Biochemistry 45 (2006), 51-60. 
[25] W. Laan, M.A. van der Horst, I.H. van Stokkum and K.J. Hellingwerf, Initial characterization of the primary photochemistry of AppA, a blue-light-using flavin adenine dinucleotide-domain containing transcriptional antirepressor protein from Rhodobacter sphaeroides: a key role for reversible intramolecular proton transfer from the flavin adenine dinucleotide chromophore to a conserved tyrosine?, Photochem. Photobiol. 78 (2003), 290-297.

[26] J. Li, Z. Liu, C. Tan, X. Guo, L. Wang, A. Sancar and D. Zhong, Dynamics and mechanism of repair of ultraviolet-induced (6-4) photoproduct by photolyase, Nature 466 (2010), 887-890.

[27] J. Li, T. Uchida, T. Ohta, T. Todo and T. Kitagawa, Characteristic structure and environment in FAD cofactor of (6-4) photolyase along function revealed by resonance Raman spectroscopy, J. Phys. Chem. B 110 (2006), 16724-16732.

[28] J. Li, T. Uchida, T. Todo and T. Kitagawa, Similarities and differences between cyclobutane pyrimidine dimer photolyase and (6-4) photolyase as revealed by resonance Raman spectroscopy: Electron transfer from the FAD cofactor to ultravioletdamaged DNA, J. Biol. Chem. 281 (2006), 25551-25559.

[29] C. Lin, D.E. Robertson, M. Ahmad, A.A. Raibekas, M.S. Jorns, P.L. Dutton and A.R. Cashmore, Association of flavin adenine dinucleotide with the Arabidopsis blue light receptor CRY1, Science 269 (1995), 968-970.

[30] S. Masuda, K. Hasegawa, A. Ishii and T.A. Ono, Light-induced structural changes in a putative blue-light receptor with a novel FAD binding fold sensor of blue-light using FAD (BLUF); Slr1694 of synechocystis sp. PCC6803, Biochemistry 43 (2004), 5304-5313.

[31] S. Masuda, K. Hasegawa, H. Ohta and T.A. Ono, Crucial role in light signal transduction for the conserved Met93 of the BLUF protein PixD/Slr1694, Plant Cell Physiol. 49 (2008), 1600-1606.

[32] S. Masuda, K. Hasegawa and T.A. Ono, Tryptophan at position 104 is involved in transforming light signal into changes of beta-sheet structure for the signaling state in the BLUF domain of AppA, Plant Cell Physiol. 46 (2005), 1894-1901.

[33] S. Masuda, K. Hasegawa and T.A. Ono, Light-induced structural changes of apoprotein and chromophore in the sensor of blue light using FAD (BLUF) domain of AppA for a signaling state, Biochemistry 44 (2005), 1215-1224.

[34] M.J. Maul, T.R. Barends, A.F. Glas, M.J. Cryle, T. Domratcheva, S. Schneider, I. Schlichting and T. Carell, Crystal structure and mechanism of a DNA (6-4) photolyase, Angew. Chem. Int. Ed. Engl. 47 (2008), 10076-10080.

[35] A. Mees, T. Klar, P. Gnau, U. Hennecke, A.P. Eker, T. Carell and L.O. Essen, Crystal structure of a photolyase bound to a CPD-like DNA lesion after in situ repair, Science 306 (2004), 1789-1793.

[36] R. Miura, Versatility and specificity in flavoenzymes: control mechanisms of flavin reactivity, Chem. Rec. 1 (2001), 183194.

[37] A.K. Murphy, M. Tammaro, F. Cortazar, Y.M. Gindt and J.P. Schelvis, Effect of the cyclobutane cytidine dimer on the properties of Escherichia coli DNA photolyase, J. Phys. Chem. B 112 (2008), 15217-15226.

[38] D. Nozaki, T. Iwata, T. Ishikawa, T. Todo, S. Tokutomi and H. Kandori, Role of Gln1029 in the photoactivation processes of the LOV2 domain in Adiantum phytochrome3, Biochemistry 43 (2004), 8373-8379.

[39] K. Okajima, Y. Fukushima, H. Suzuki, A. Kita, Y. Ochiai, M. Katayama, Y. Shibata, K. Miki, T. Noguchi, S. Itoh and M. Ikeuchi, Fate determination of the flavin photoreceptions in the cyanobacterial blue light receptor TePixD (T110078), J. Mol. Biol. 363 (2006), 10-18.

[40] A. Pfeifer, T. Majerus, K. Zikihara, D. Matsuoka, S. Tokutomi, J. Heberle and T. Kottke, Time-resolved Fourier transform infrared study on photoadduct formation and secondary structural changes within the phototropin LOV domain, Biophys. J. 96 (2009), 1462-1470.

[41] A. Pfeifer, T. Mathes, Y. Lu, P. Hegemann and T. Kottke, Blue light induces global and localized conformational changes in the kinase domain of full-length phototropin, Biochemistry 49 (2010), 1024-1032.

[42] M. Salomon, J.M. Christie, E. Knieb, U. Lempert and W.R. Briggs, Photochemical and mutational analysis of the FMNbinding domains of the plant blue light receptor, phototropin, Biochemistry 39 (2000), 9401-9410.

[43] Y. Sato, T. Iwata, S. Tokutomi and H. Kandori, Reactive cysteine is protonated in the triplet excited state of the LOV2 domain in Adiantum phytochrome3, J. Am. Chem. Soc. 127 (2005), 1088-1089.

[44] Y. Sato, M. Nabeno, T. Iwata, S. Tokutomi, M. Sakurai and H. Kandori, Heterogeneous environment of the S-H group of Cys966 near the flavin chromophore in the LOV2 domain of Adiantum neochrome1, Biochemistry 46 (2007), 1025810265.

[45] J.P.M. Schelvis, M. Ramsey, O. Sokolova, C. Tavares, C. Cecala, K. Connell, S. Wagner and Y.M. Gindt, Resonance Raman and UV-Vis spectroscopic characterization of FADH(center dot) in the complex of photolyase with UV-damaged DNA, J. Phys. Chem. B 107 (2003), 12352-12362.

[46] E. Schleicher, B. Hessling, V. Illarionova, A. Bacher, S. Weber, G. Richter and K. Gerwert, Light-induced reactions of Escherichia coli DNA photolyase monitored by Fourier transform infrared spectroscopy, FEBS J. 272 (2005), 1855-1866.

[47] T. Senda, M. Senda, S. Kimura and T. Ishida, Redox control of protein conformation in flavoproteins, Antioxid. Redox Signal. 11 (2009), 1741-1766.

[48] K. Sokolowsky, M. Newton, C. Lucero, B. Wertheim, J. Freedman, F. Cortazar, J. Czochor, J.P. Schelvis and Y.M. Gindt, Spectroscopic and thermodynamic comparisons of Escherichia coli DNA photolyase and Vibrio cholerae cryptochrome 1, J. Phys. Chem. B 114 (2010), 7121-7130. 
[49] A.L. Stelling, K.L. Ronayne, J. Nappa, P.J. Tonge and S.R. Meech, Ultrafast structural dynamics in BLUF domains: transient infrared spectroscopy of AppA and its mutants, J. Am. Chem. Soc. 129 (2007), 15556-15564.

[50] H. Suzuki, K. Okajima, M. Ikeuchi and T. Noguchi, LOV-like flavin-Cys adduct formation by introducing a Cys residue in the BLUF domain of TePixD, J. Am. Chem. Soc. 130 (2008), 12884-12885.

[51] T.E. Swartz, P.J. Wenzel, S.B. Corchnoy, W.R. Briggs and R.A. Bogomolni, Vibration spectroscopy reveals light-induced chromophore and protein structural changes in the LOV2 domain of the plant blue-light receptor phototropin 1, Biochemistry 41 (2002), 7183-7189.

[52] T. Todo, H. Ryo, K. Yamamoto, H. Toh, T. Inui, H. Ayaki, T. Nomura and M. Ikenaga, Similarity among the Drosophila (6-4)photolyase, a human photolyase homolog, and the DNA photolyase-blue-light photoreceptor family, Science 272 (1996), 109-112.

[53] M. Unno, S. Kikuchi and S. Masuda, Structural refinement of a key tryptophan residue in the BLUF photoreceptor AppA by ultraviolet resonance Raman spectroscopy, Biophys. J. 98 (2010), 1949-1956.

[54] M. Unno, S. Masuda, T.A. Ono and S. Yamauchi, Orientation of a key glutamine residue in the BLUF domain from AppA revealed by mutagenesis, spectroscopy, and quantum chemical calculations, J. Am. Chem. Soc. 128 (2006), 5638-5639.

[55] M. Unno, R. Sano, S. Masuda, T.A. Ono and S. Yamauchi, Light-induced structural changes in the active site of the BLUF domain in AppA by Raman spectroscopy, J. Phys. Chem. B 109 (2005), 12620-12626.

[56] A. Yamamoto, T. Iwata, Y. Sato, D. Matsuoka, S. Tokutomi and H. Kandori, Light signal transduction pathway from flavin chromophore to the J alpha helix of Arabidopsis phototropin1, Biophys. J. 96 (2009), 2771-2778.

[57] A. Yamamoto, T. Iwata, S. Tokutomi and H. Kandori, Role of Phe1010 in light-induced structural changes of the neo1LOV2 domain of Adiantum, Biochemistry 47 (2008), 922-928.

[58] Y. Zhang, T. Iwata, J. Yamamoto, K. Hitomi, S. Iwai, T. Todo, E.D. Getzoff and H. Kandori, FTIR study of light-dependent activation and DNA repair processes of (6-4) photolyase, Biochemistry 50 (2011), 3591-3598. 


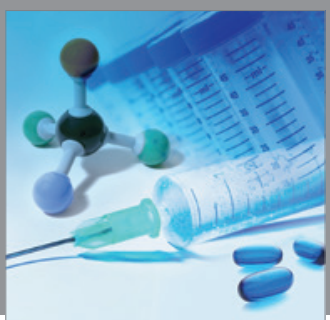

International Journal of

Medicinal Chemistry

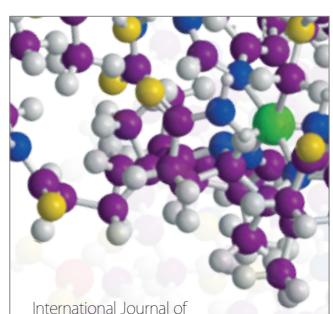

Carbohydrate Chemistry

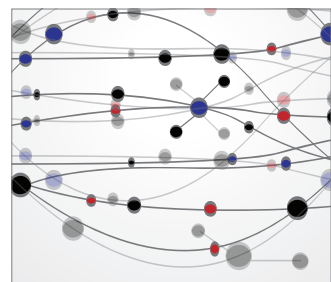

The Scientific World Journal
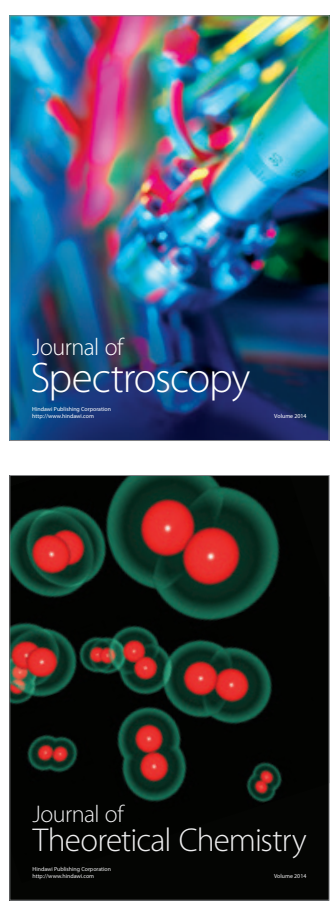
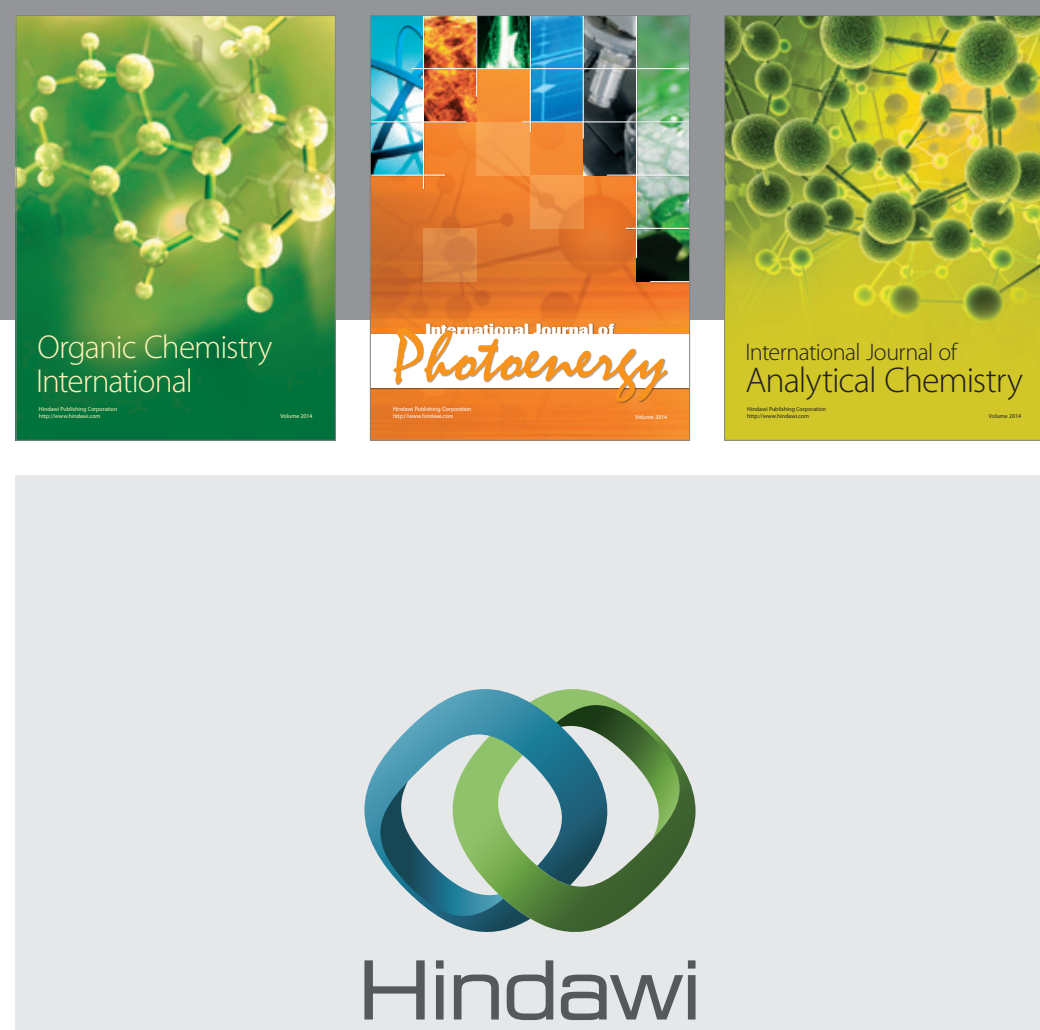

Submit your manuscripts at

http://www.hindawi.com
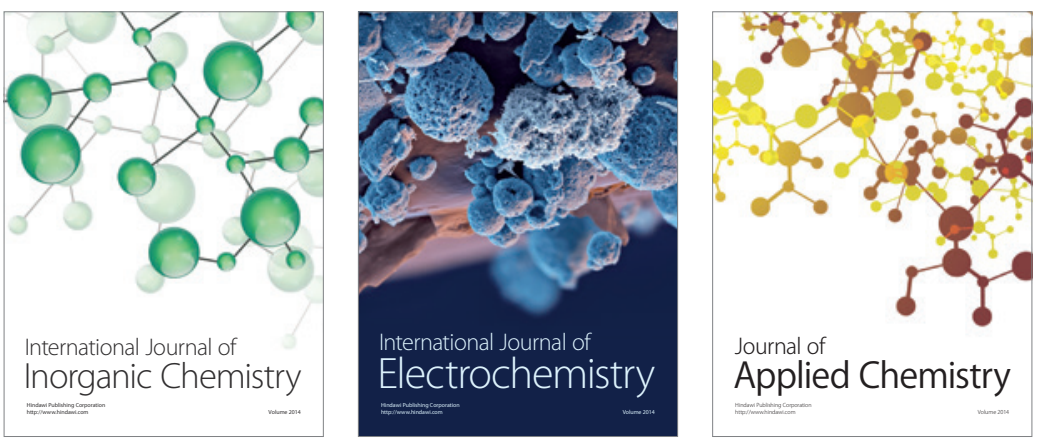

Journal of

Applied Chemistry
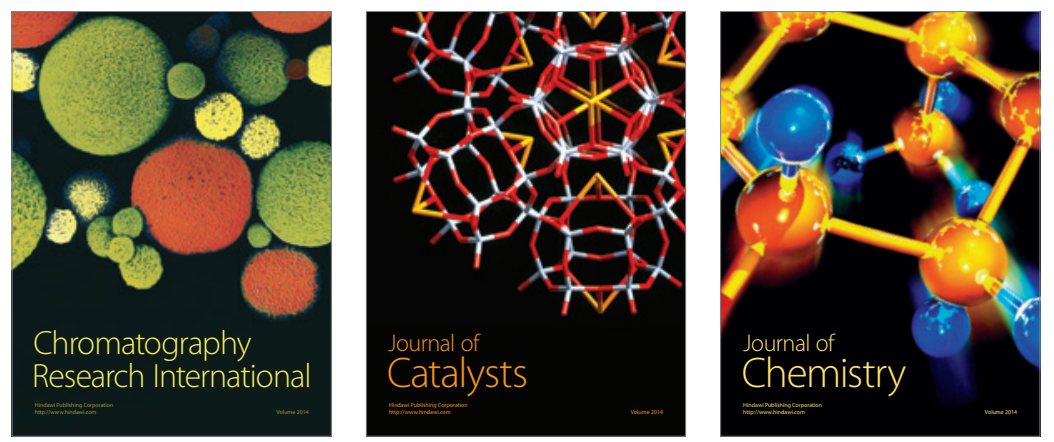
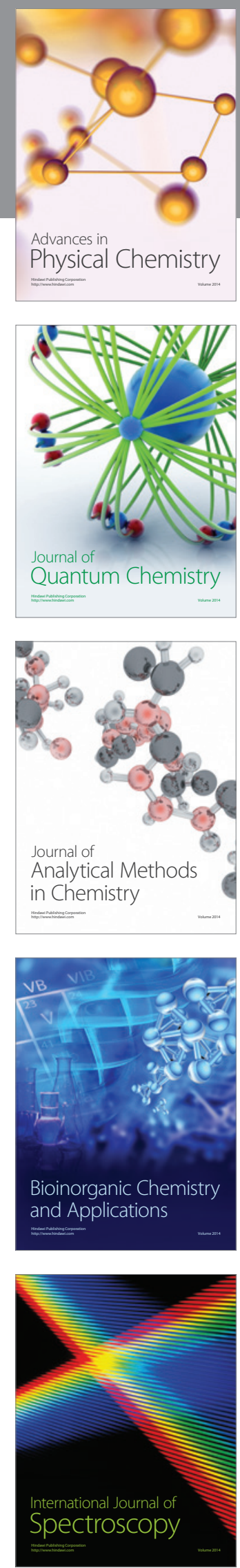